\title{
ANO6 promotes cell proliferation and invasion in glioma through regulating the ERK signaling pathway
}

This article was published in the following Dove Press journal: OncoTargets and Therapy

\author{
Zhao-bo Xuan ${ }^{1, *}$ \\ Ye-ji Wang ${ }^{2, *}$ \\ Jun $\mathrm{Xie}^{3}$
}

'Department of Neurosurgery, The First Hospital Affiliated to Jiamusi University, Jiamusi City, Heilongjiang Province I54002, People's Republic of China; ${ }^{2}$ Department of Neurosurgery, Shanxian Haijiya Hospital, Heze City, Shandong Province 274300, People's Republic of China; ${ }^{3}$ Department of Neurosurgery, Tongchuan People's Hospital, Tongchuan City, Shaanxi Province 727000, People's Republic of China

*These authors contributed equally to this work
Correspondence: Jun Xie

Tongchuan People's Hospital, No. 12,

Health Road, Wangyi District, Tongchuan

City, Shaanxi Province 727000, People's

Republic of China

$\mathrm{Tel} / \mathrm{Fax}+869192827120$

Email JunXie8989@I63.com
Purpose: Anoctamin6 (ANO6) plays a crucial role in several cancers, whereas the specific role of ANO6 in glioblastoma is unclear.

Methods: Kaplan-Meier survival analysis was used to analysis the correlation between ANO6 and survival rate of patients with glioblastoma. Univariate Cox regression analysis was used to analysis the correlation among ANO6 expression level,and age, gender, WHO and overall survival rate. Immunohistocemical technique, RT-PCR and western blot were used to dected the ANO6 expression. CCK8, colony formation and transwell were used to detected cell viability, cell proliferation and cell invasion in glioblastoma cells transfected with sh-ANO6 and ANO6 overexpression. In addition, after SHG-44 cells trasfected with ANO6 overexpression were ERK inhibitor (PD98059), CCK8, colony formation and transwell were used to detected cell viability, cell proliferation and cell invasion. Western blot was used to detected ERK protein level and the phosphorylation level of ERK in T89G and U87MG cells tranfected wih sh-ANO6. Results: The results indicated that the ANO6 expression level was significantly associated with patients' age and tumor stage. Univariate Cox regression analysis showed that the ANO6 expression level, age, gender and tumor stage were not related to the overall survival rate. ANO6 inhibition significantly suppressed the viability, invasion and the ability of colony formation in glioma cells, while ANO6 overexpression led to the opposite results in SHG-44 cells. ANO6 knockdown strongly inhibits the phosphorylation level and nuclear translocation of extracellular signal-regulated kinase (ERK) protein to inhibit ERK signaling. ERK inhibitor significantly decreased the cell proliferation and invasion in SHG-44 cells transfected with sh-ANO6.

Conclusion: This study revealed that ANO6 activited ERK signaling pathway through promoting the nuclear translocation of ERK to increase the proliferation and invasion of glioblastoma cells

Keywords: ANO6, proliferation, invasion, ERK signaling pathway, glioblastoma

\section{Introduction}

Glioma is the most common primary malignant tumor in the central nervous system, which is characterized by the high morbidity, high malignant grade and poor therapeutic effect. The biological behavior of glioma manifests as infiltrative growth, with no obvious boundary from the normal brain tissue. ${ }^{1}$ The World Health Organization (WHO) has classified glioma into 4 grades according to the histological standard, namely, WHO I, II, III and IV. Among them, gliomas at WHO I and WHO II grades grow at a low rate with favorable prognosis, while those at WHO III and WHO IV grades are highly invasive with poor prognosis. ${ }^{2,3}$ Research 
indicates that the median survival for glioma patients is about 14-16 months after combined surgery, chemotherapy and radiotherapy. ${ }^{4,5}$ Therefore, it is urgent to search for a new sally port for the early diagnosis and targeted treatment of glioma.

The Anoctamin (ANO) family has contained 10 different proteins, including ANO1 - ANO9. ${ }^{6}$ Research indicates that Anoctamin exists in the form of dimer, and its subunit contains 10 transmembrane domains as well as the calcium ion $(\mathrm{Ca} 2+)$-binding site located in the hydrophobic hole. ${ }^{7}$ ANO6 (called TMEM16F) is the homologous transmembrane protein that shares numerous similar sequences in the transmembrane structure with ANO1. ${ }^{7}$ Recent research discovers that ANO6 plays a key role in the $\mathrm{Ca} 2+$-dependent phospholipid switching process. ${ }^{8}$ Scott syndrome patients will develop homozygous mutation of the ANO6 gene, which would result in the early termination of the translation process. ${ }^{9}$ Meanwhile, ANO1, ANO3, ANO5 and ANO6 mRNA levels are elevated in skeletal development. ${ }^{10-12}$ ANO6 can serve as a kind of osmoreceptor to participate in regulating the cell defense capacity. Some research suggests that some Anoctamin are slightly upregulated in tumor, while the phospholipid rearrangement on membrane is related to cell migration, and suppressing the activity of the phospholipid flippase can result in the declined cell migration capacity. ${ }^{13,14}$ Jacobsen et al had discovered when studying the effects of ANO1 and ANO6 on cell migration that ANO1 knockout would affect the cell migration direction, while ANO6 knockout would decrease the cell migration rate. ${ }^{15}$ ANO1 expression was found to correlate with greater tumor size, increased ERK $1 / 2$ activity to inhibit cell apoptosis in human head and neck squamous cell carcinomas. ${ }^{16}$ These findings revealed that ANO6 inhibition or/overexpression may partially affect tumor metastasis and be closely related to ERK signaling activation, which has brought a new thinking for tumor treatment.

The signal transduction pathway of ERK, the extracellular signal-regulating kinase, starts with the signals transmitted by protein kinase C or Ras; afterward, the Raf can be activated to initiate the cascade reaction including mitogen-activated protein kinase kinase 7 (MEK) and ERK activation. ${ }^{17}$ MEK can phosphorylate threonine-x-tyrosine (TXY) in the enzymatic region of ERK, thus activating the core of the entire ERK signaling pathway downstream. The activated ERK can promote target protein phosphorylation within the cytoplasm or regulate the activity of other protein kinase; at the same time, it can phosphorylate the cytoskeleton component, regulate cell morphology and skeletal redistribution. ${ }^{18}$ When the activated ERK enters the cell nucleus, it can regulate gene expression through the phosphorylated transcription factor, thus participating in regulating cell proliferation and differentiation. ${ }^{19}$

In this study, CCK-8, immunohistochemistry, RT-PCR, Western blotting, Transwell assay, colony formation assay and cell transfection assay were performed, so as to detect the effects of ANO6 on the proliferation and invasion of glioma cells in vitro, and to explore the mechanism of action of ANO6. Hopefully, this study could provide theoretical foundation for the treatment of glioma via exploring the role of ANO6 in the proliferation and invasion of glioma.

\section{Materials and methods}

\section{Tissues samples}

Glioma and adjacent normal tissue specimens of 43 patients with glioblastoma (mean age, $43.2 \pm 10.4$ years; 22 males and 21 females) were obtained from January 2016 to December 2018 at The First Hospital Affiliated to Jiamusi University. The following eligibility criteria were included in our study: (1) the histopathological classification and grading of glioma met WHO criteria; (2) surgical extraction of tumor lesions; (3) no preoperative anti-cancer treatment such as chemotherapy and radiotherapy; (4) complete postoperative follow-up data and (5) no other primary or familial malignancies. All patients underwent TNM staging according to the 7th edition of AJCC system. Overall survival (OS) was defined as death from any cause from the time of surgery. The patients' clinical information is listed in Table 1. All cases were reviewed

Table I Correlations between ANO6 expression in glioma and clinical characteristics

\begin{tabular}{|c|c|c|c|c|}
\hline \multirow[t]{2}{*}{ Group } & \multirow[t]{2}{*}{ No. } & \multicolumn{2}{|c|}{ Relative ANO6 expression } & \multirow[t]{2}{*}{$p$-Value } \\
\hline & & Low & High & \\
\hline \multicolumn{5}{|l|}{ Gender } \\
\hline Male & 22 & 11 & 11 & 0.432 \\
\hline Female & 21 & 8 & 13 & \\
\hline \multicolumn{5}{|l|}{ Age } \\
\hline$\leq 40$ & 17 & 14 & 3 & $0.000 *$ \\
\hline$>40$ & 26 & 5 & 21 & \\
\hline \multicolumn{5}{|l|}{ WHO grade } \\
\hline I-II stage & 34 & 17 & 17 & $0.028 *$ \\
\hline III-IV stage & 9 & 2 & 7 & \\
\hline
\end{tabular}

Notes: $\chi^{2}$ test, $* P<0.05$. 
by pathologist and histologically confirmed as pancreatic cancer based on histopathological evaluation. All tissues were immediately snap-frozen in liquid nitrogen and stored at $-80^{\circ} \mathrm{C}$ until further experimentation. Studies were approved by the Ethics Committee of The First Hospital Affiliated to Jiamusi University, and all of the samples were obtained with written informed consent and analyzed anonymously. The study was conducted in accordance with the Declaration of Helsinki.

\section{Cell culture}

Four golima cell lines (T98G, U87MG, SHG-44 and SWO-38) and normal human astrocytes (NHAs) were bought from American Type Culture Collection (ATCC, Manassas, VA, USA). All cells were incubated in Dulbecco's modified Eagle's medium (DMEM) (Invitrogen, Carlsbad, CA, USA) containing 10\% FBS (Gibco, Grand Island, NY, USA), $100 \mathrm{U} / \mathrm{mL}$ penicillin, as well as $100 \mu \mathrm{g} / \mathrm{mL}$ streptomycin, which were cultured in humidified chamber at $37^{\circ} \mathrm{C}$ containing $5 \% \mathrm{CO}_{2}$.

\section{Immunohistochemistry assay}

For immunohistochemical staining, the paraffin-embedded golima tissues were deparaffinized in xylene, rehydrated and washed in PBS. After antigen retrieval, the samples were blocked and incubated with primary antibody against ANO6 (1:500, Cat\#PA5-58,610, ThermoFisher, California, USA) at $4{ }^{\circ} \mathrm{C}$ overnight. After being rinsed with PBS three times for 5 mins each, sections were treated with biotinylated secondary antibody (Abcam, Cambridge, USA) for 1 $\mathrm{hr}$, followed by incubation with streptavidin-horseradish peroxidase (HRP) for 20 mins. Diaminobenzidine (DAB) substrate was used as color-developing agent for visualization of ANO6-positive cells. The expression level of ANO6 was independently evaluated by 2 pathologists who were blind to the clinical data, according to the proportion and intensity of positive cells that were determined within 5 microscopic visual fields per slide. In brief, for quantitative scoring analysis, the percentage of positive-stained cells (PP) was categorized as assigned to the one of five categories: score $0,0-9 \%$; score 1, 10-25\%; score 2, 26-50\%; score 3, 51-75\%; score 4, 76-100\%. The staining intensity (SI) was independently categorized as no staining (score 0 ), weak (score 1), moderate (score 2) or strong (score 3 ). The total score was calculated according to the immunoreactive score (IRS): IRS $=\mathrm{SI} \times \mathrm{PP}$, ranging from 0 to 12 .

\section{ANO6 gene silencing}

Transfection of T98G and U87MG cells (60-70\% confluence) was performed with siRNAs against ANO6 or negative control oligos (Ribobio, Guangzhou, China) were labeled as ANO6-knockdown (KD)1, ANO6-KD2 or NC, respectively. The sequences of ANO6 silencing were as follows (ANO6-KD1): sense, 5'-GCATACGAATCTAAC CTTATCCGAAGATAAGGTTAGATTCGTATGC-3' and antisense, 5'-GCATACGAATCTAACCTTATCTTCGGAT AAGGTTAGATTCGTATGC-3'. The sequences of ANO6 silencing were as follows (ANO6-KD2): sense, 5'-GCT CATCCTCGAAGCATATACTCAAGAGGTATATGCTTCGAGGATGAGC-3' and antisense, 5'-GCTCATCCTCG AAGCATATACCTCTTGAGTATATGCTTCGAGGATGAGC-3'. Lipofectamine 2000 (Invitrogen) was used for Oligonucleotide transfection according to the manufacturers' recommendations.

\section{Lentivirus production and transfection}

The lentiviruses included ANO6-OE (ANO6-overexpressing lentivirus) and CTRL (blank lentivirus) plasmids, which were designed and generated by GENECHEM (Shanghai, China). According to the manufacturer's instruction, the lentiviruses $\left(10^{8} \mathrm{TU} / \mathrm{mL}\right)$ were used to transfect the cells, and puromycin $(1.0 \mu \mathrm{g} / \mathrm{m})$ was applied to collect single clones showing infection efficiency $\geq 80 \%$ and good growth status by microscopic observation. The sequences of overexpressed ANO6 were as follows: sense, 5'-ATGAAAAAGATGAGCAGGAATG-3' and antisense, 5'-TTATTCTGATTTTGGCCGTA-3'. ANO6 expression was confirmed by RT-PCR, and the levels of ANO6 protein were measured by Western blotting.

\section{RNA isolation and RT-PCR}

RNA was isolated from glioblastoma tissues and cell lines using TRIzol ${ }^{\circledR}$ reagent (Invitrogen, Carlsbad, CA, USA) in line with the manufacturer's protocol. cDNA preparation was performed with Revert Aid Kit purchased from Thermo Fisher Scientific (Waltham, MA). The cDNA probes were synthesized from one microgram of total RNA. RT-PCR was performed with DreamTaq Green PCR Master Mix (ThermoFisher Scientific, Waltham, MA) by adding $1 \mu \mathrm{L}$ primer, $0.5 \mu \mathrm{L}$ ( $\beta$-actin primer) and $2 \mu \mathrm{L}$ (Dkk1 primer) of cDNA per $20-\mu \mathrm{L}$ reaction, respectively. Gel electrophoresis for fractionation of the nuclear acids was performed with $1.5 \%$ agarose gels. qRT-PCR was performed in $20-\mu \mathrm{L}$ reaction, $1 \mu \mathrm{L}$ primer and $1 \mu \mathrm{L}$ cDNA were added to the 
fluoresced SYBR-Green Master-Mix (Thermo Fisher Scientific) and gene expression was analyzed with RotorGene Analysis Software (Qiagen Corbett Research). Relative quantification to reference gene $\beta$-actin was calculated with the $\Delta \Delta \mathrm{Ct}$-method. The primers used as follows: for ANO6 [Forward- TCTACAAGGCAGCTTTCCCA, Reverse- GTGTAATAGCCCAGCCAAGC] and $\beta$-actin [Forward-CACTCTTCCAGCCTTCCTTC, Reverse-GGT GTAACGCAACTAAGTCATAG]. ${ }^{20}$

\section{MTT assay}

MTT assay was used to detect cell viability in each group. Cells were seeded into 96-well plates at a density of 1000 cells/well and cultured in $100 \mu \mathrm{L}$ of DMEM containing $10 \%$ FBS for 4 days. At each indicated time point, $20 \mu \mathrm{L}$ of MTT (Beyotime, Jiangsu, China) solution was added to each corresponding well and incubated for $4 \mathrm{hrs}$. Formazan crystals were then solubilized with $150 \mu \mathrm{L}$ dimethyl sulfoxide (Beyotime) and the absorbance value (OD) was measured at $450 \mathrm{~nm}$ using a microplate reader.

\section{Western blotting}

Total proteins were extracted with RIPA lysis buffer supplemented with protease inhibitors, separated by SDSPAGE, and transferred to a PVDF membrane (Millipore, Billerica, MA). The membrane was blocked with $5 \%$ non-fat milk at room temperature for $1 \mathrm{hr}$ and incubated with the appropriate antibody. Protein bands were visualized using the chemiluminescent HRP detection system (Millipore). The antibodies are as follows: ANO6 (1:1000, cat\#PA5-69345, ThermoFisher, California, USA), ERK (1:1000, cat\#13-6200, ThermoFisher, California, USA), phosphorylation (phospho)-ERK (1:500, cat\#44680G, ThermoFisher, California, USA), LaminB (1:2000, cat\#PA5-66473, ThermoFisher, California, USA), $\beta$-actin (1:5000, cat\#MA5-15739, ThermoFisher, California, USA).

\section{Colony formation assay}

Cells were plated in 6-well culture plates at 100 cells/ well. After an incubation for 14 days at $37^{\circ} \mathrm{C}$, the cells were washed twice with PBS and stained with $0.1 \%$ crystal violet solution (Beyotime). The number of colonies containing $\geq 50$ cells was counted under a microscope. The formula for colony formation efficiency was as follows: (number of colonies/number of cells inoculated) $\times 100 \%$.

\section{Transwell invasion assays}

The abilities of invasion in each group cell were detected by using Transwell assay as described in the previous steps. ${ }^{21}$ Briefly, cells were plated within the top chamber coated with Matrigel membrane at a density of $3 \times 106$ cells/well. 10\% FBS in the lower chamber was used as a chemoattractant. Cells were incubated for $48 \mathrm{hrs}$ and the cells that did not invade through the membrane were removed by a cotton swab. Cells on the lower chamber were then stained with $0.1 \%$ crystal violet (Beyotime) and counted in 4 microscopic visual fields per slide under an invert microscope (Olympics).

\section{Statistical analysis}

In this study, we defined the relative ANO6 expression $>8$ as high expression and 8 is derived from the mean values of all samples. Survival analysis was compared using the univariate Cox proportional hazard model among different ANO6 mRNA expression levels in glioma tissue. The correlations between ANO6 expression and clinicopathological characteristics were examined by $\chi^{2}$ test. The results presented as mean $\pm \mathrm{SD}$ were analyzed with a two-sided Student's $t$-test for two groups and one-way ANOVA test for three or more groups. $p<0 \quad 0.05$ was considered as statistically significant. Survival curves were constructed using the Kaplan-Meier method and analyzed by the log-rank test.

\section{Results}

\section{Expression level of ANO6 was} significantly increased in glioma tissues and cell lines and associated with WHO grade and poor prognosis

The expression level of ANO6 in glioma tissue specimens $(\mathrm{n}=43$; mean age, $43.2 \pm 10.4$ years; 22 males and 21 females) and matched adjacent normal tissues was analyzed by RT-PCR, Western blot and immunohistochemical assays. Results showed that the expression level of ANO6 in glioma tissues was significantly higher than in the adjacent normal glioma tissues (Figure $1 \mathrm{~A}-\mathrm{C}$ ). We also found that ANO6 expression correlated with WHO grade and patients' age $(p<0.05$; Table 1$)$. However, there was no significant correlation with patient gender (Table 1). In addition, Kaplan-Meier analysis revealed that patients with high ANO6 expression (we defined the relative expression $>8$ as high expression) clearly had poorer tumor-free 
A

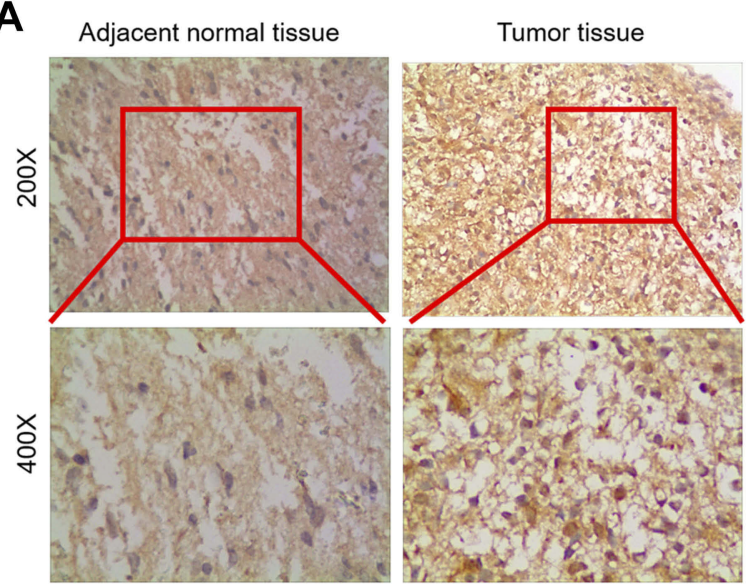

B
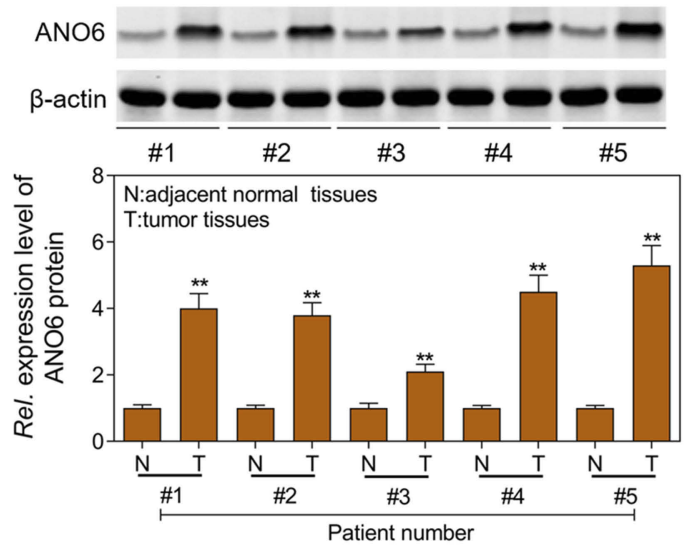

C

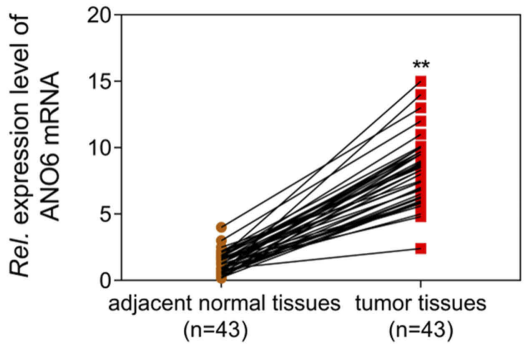

D

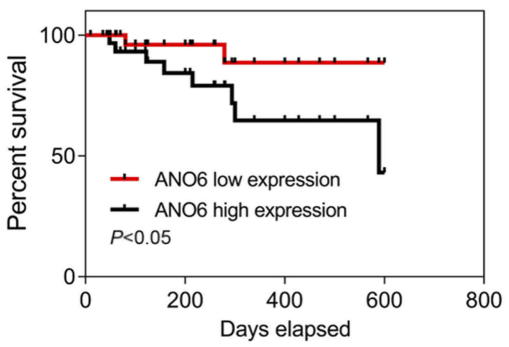

E
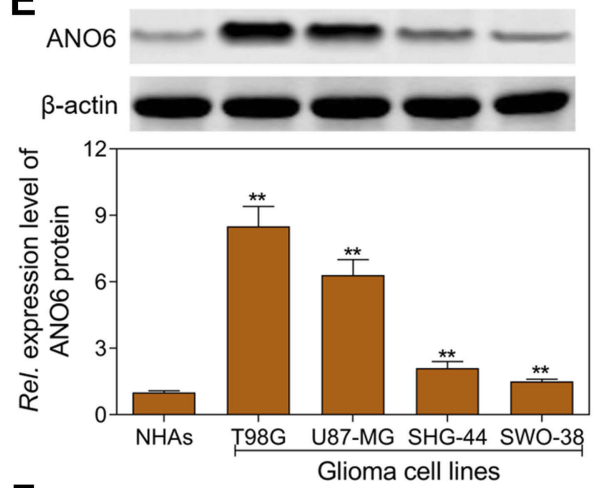

F

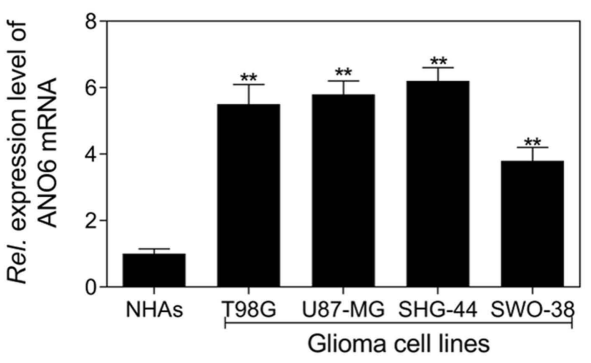

G

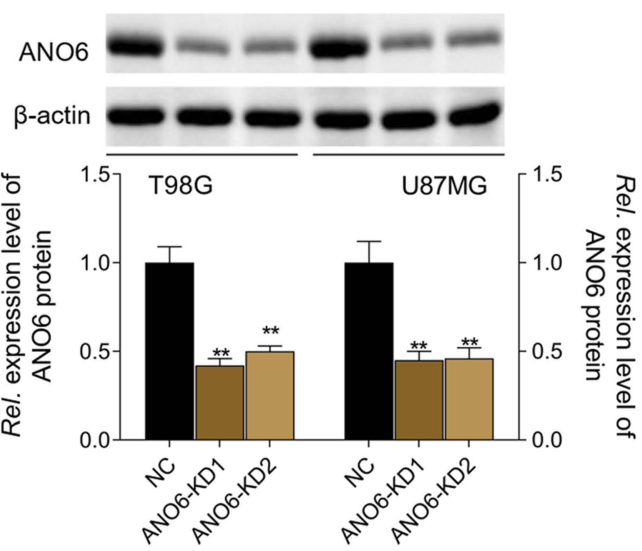

H

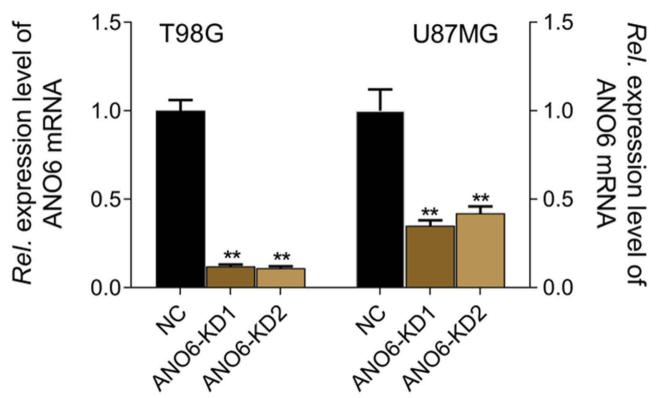

Figure I Expression level of ANO6 was significantly increased in glioma tissues and cell lines and associated with poor prognosis. (A) ANO6 expression in glioma tissues and normal tissues of 6 selected patients was examined by immunohistochemical analysis; (B) the protein expression level of ANO6 gene was detected by Western blot assay in glioma tissues and normal tissues of 5 patients; (C) the mRNA expression level of ANO6 gene was detected by RT-PCR in glioma cell lines in glioma tissues and normal tissues of 43 patients; (D) Kaplan-Meier survival analysis was performed to investigate the relationship between ANO6 low expression and ANO6 high expression; (E) the protein expression level of ANO6 gene was detected by Western blot assay in glioma cell lines; (F) the mRNA expression level of ANO6 gene was detected by RTPCR assay in glioma cell lines; $(\mathbf{G})$ the protein expression level of ANO6 gene was detected by Western blot assay in T98G and U87MG cells transfection with ANO6 KDI/ or 2; $\mathbf{( H )}$ the mRNA expression level of ANO6 gene was detected by RT-PCR assay in T98G and U87MG cells transfection with ANO6 KDI/or 2. $\beta$-actin was used as a load control. Data are presented as the mean \pm standard deviation. ${ }^{* *} p<0.05$ versus Normal group/or negative control group, and Rel. is relative. 
Table 2 Univariate Cox proportional hazards analyses of ANO6 expression and overall survival for patients with glioma

\begin{tabular}{|c|c|c|}
\hline & \multicolumn{2}{|l|}{ Univariate analysis } \\
\hline & HR (95\% Cl) & $P$-value \\
\hline \multicolumn{3}{|l|}{ Gender } \\
\hline Male vs female & $0.998(0.504-1.976)$ & 0.996 \\
\hline \multicolumn{3}{|l|}{ Age } \\
\hline$\leq 40$ vs $>40$ & $0.998(0.501-1.991)$ & 0.996 \\
\hline \multicolumn{3}{|l|}{ WHO stage } \\
\hline | stage vs || stage & $0.997(0.452-2.197)$ & 0.994 \\
\hline II stage vs III stage & $1.000(0.39 I-2.556)$ & 1.000 \\
\hline III stage vs IV stage & $1.000(0.133-7.540)$ & 1.000 \\
\hline \multicolumn{3}{|l|}{ ANO6 expression } \\
\hline High vs Low & I.I27 (0.569-2.230) & 0.732 \\
\hline
\end{tabular}

Note: $P<0.05$.

Abbreviations: $\mathrm{HR}$, Hazard ratio; $\mathrm{Cl}$, confidence interval.

survival (Figure 1D). We used univariate Cox regression to analyze the possible prognostic factors in postoperative patients with glioma, and they are listed in Table 2 . Univariate Cox regression analysis showed that the ANO6 expression level, age, gender and WHO stage were not related to the OS rate. Next, the expression level of ANO6 was measured in the glioma cell lines: T98G, U87-MG, SHG-44 and SWO-38. Compared with the normal human astrocytes cell line, the protein and mRNA expression levels of ANO6 were upregulated in T98G, U87-MG, SHG-44 and SWO-38 cells, particularly in T98G and U87-MG cells (Figure 1E and F).

\section{Knockdown of ANO6 inhibited the proliferation and invasion of T98G and U87MG cells by inhibiting the nuclear translocation of ERK}

The expression levels of ANO6 mRNA and protein of ANO6-KD1 and ANO6-KD2 groups were decreased in T98 and U87MG by RT-PCR and Western blot assays (Figure $1 \mathrm{G}$ and $\mathrm{H}$ ). MTT and colony formation assays were used to detect the cell proliferation, and transwell assay was used to detect the cell invasion. These results showed that the cell proliferation of ANO6 knockdown cells notably decreased (Figure $2 \mathrm{~A}-\mathrm{D} ; p<0.05$ ), while the ability of invasion of the ANO6-KD1 and ANO6-KD2 groups was significantly decreased (Figure $2 \mathrm{E}$ and $\mathrm{F}$; $P<0.05$ ), compared with those of NC group. Moreover, we found that the phosphorylation level of ERK protein in ANO6-KD1 and ANO6-KD2 groups was significantly decreased (Figure $2 \mathrm{E}$ and $\mathrm{F} ; P<0.05$ ), compared with those of NC group. Thus, to determine whether ANO6 directly affects the ERK signaling activation in T98G and U87MG cells, Western blotting was conducted by knocking down ANO6. Our results showed that the phosphorylation level of ERK was significantly downregulated in glioma cells' transfection with ANO6 silence plasmids (Figure 3A and B). Further analysis revealed that ANO6 was significantly decreasing after cells transfection with sh-ANO6 (Figure 3C). The protein expression level of ERK in cytoplasm of T98G cell was higher than that of the nucleus of T98G and U87MG cells (Figure 3C-H). ANO6 knockdown could significantly inhibit the protein expression level of ERK in cytoplasm and nucleus of T98G and U87MG cells (Figure 3C-H). Thus, we conclude that ANO6 knockdown inhibits the nuclear translocation of ERK in glioma cells to some extent.

\section{ANO6 overexpression promoted the proliferation and invasion of SHG-44G cells}

In order to further verify the above results, ANO6 was overexpressed in SHG-44 cells to obverse the biological behaviors. MTT, colony formation and transwell assays were used to detect the proliferation and invasion of SHG-44 cells. We found that ANO6 overexpression could significantly promote the viability and the abilities of colony formation and invasion in SHG-44 cells, compared with those of CTRL group (Figure 4). There is a reverse trend in cell proliferation and invasion between ANO6 inhibition and ANO6 overexpression groups.

\section{ERK inhibitor inhibited the proliferation and invasion of ANO6 overexpression cells}

MTT results showed that ANO6 overexpression increased the viability of SHG-44 cells, while ERK inhibitor decreased the viability of ANO6 overexpression cells (Figure 5A). Further, transwell and colony formation assays results showed that the abilities of colony formation and invasion were significantly increased in SHG-44 cells, whereas, the abilities of colony formation and invasion were significantly decreased by ERK inhibitor in ANO6 overexpression cells (Figure 5B-E) 
A
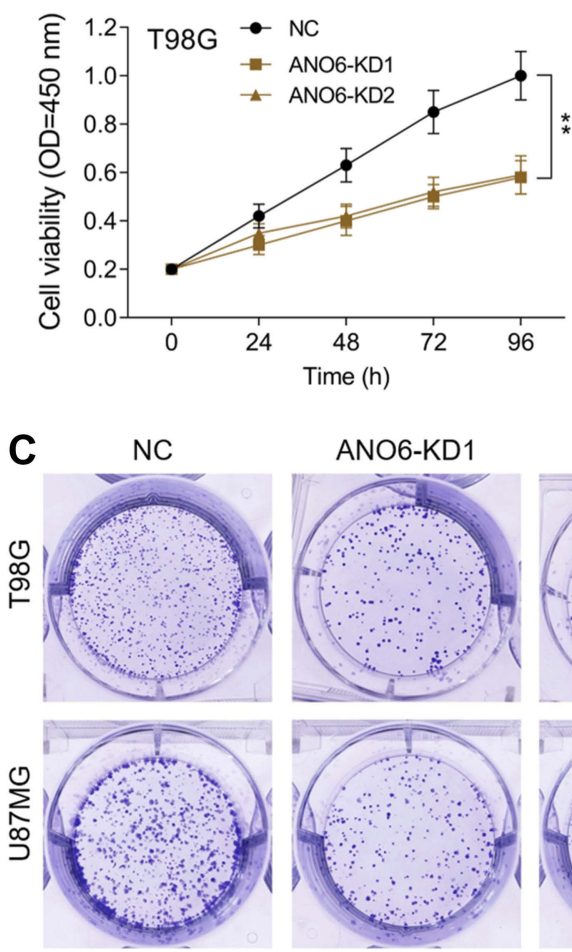

ANO6-KD1
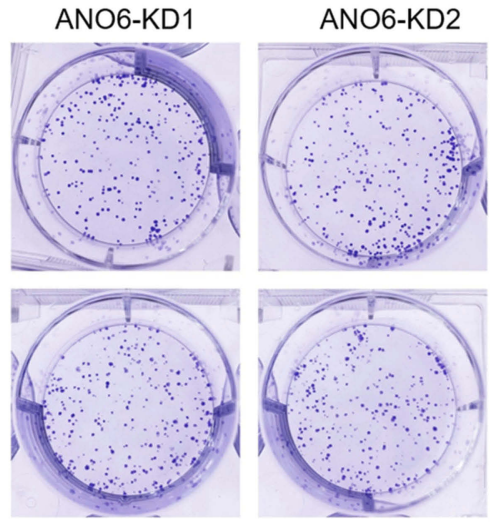

E

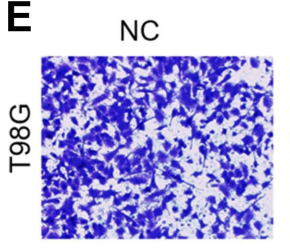

ANO6-KD1
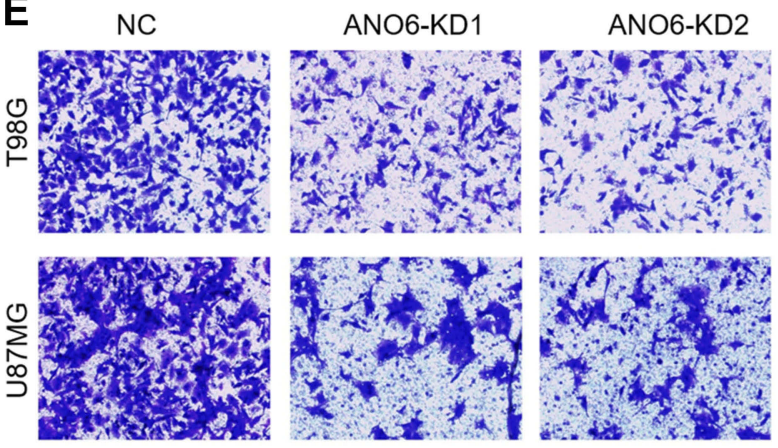

B

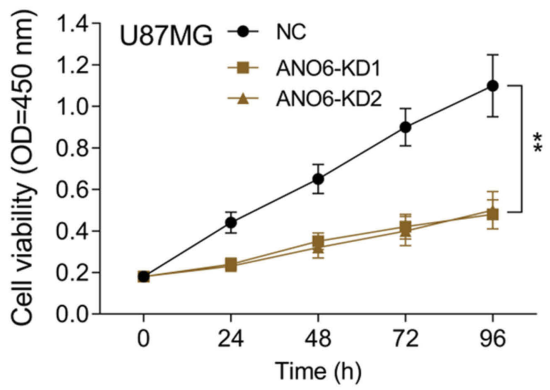

D

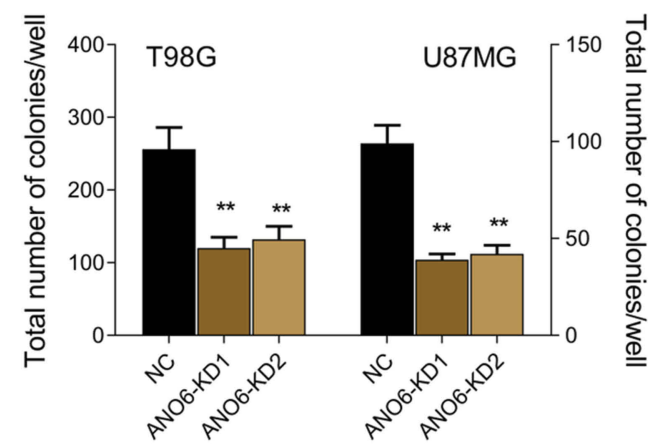

\section{$\mathbf{F}$}

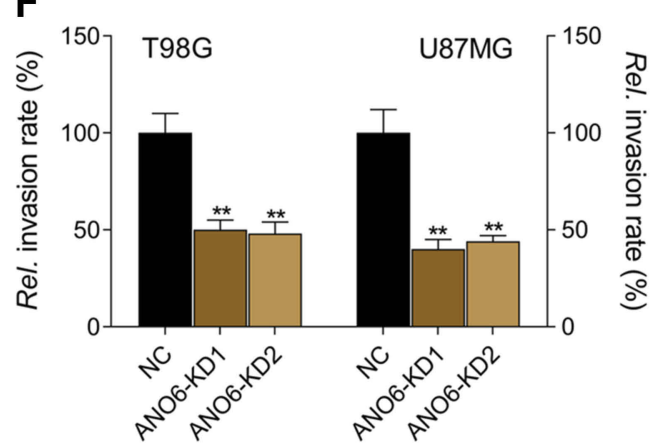

Figure 2 Knockdown of ANO6 inhibited the proliferation and invasion of T98G and U87MG cells. After T98G and U87MG cells transfection with ANO6-KD I or ANO6KD2, (A) the cell viability was detected by MTT assay in T98G cells; (B) the cell viability was detected by MTT assay in U87MG cells; (C and D) the ability of cell proliferation of T98G and U87MG cells transfection with sh-ANO6 was detected by colony formation; (E and $\mathbf{F})$ the ability of invasion of T98G and U87MG cells transfection with sh-ANO6 was detected by transwell assay. Data are presented as the mean \pm standard deviation. **p<0.05 versus ANO6-KDI group and ANO6-KD2 group. Abbreviation: NC, negative control.

\section{Discussion}

ANO6 is reported to be a member of a family of transmembrane proteins expressed in a variety of cells..$^{7,15,22,23}$ In recent years, the research of ANO6 has become increasingly intensive because of its wide distribution, versatility and its close relationship with the occurrence and development of various diseases. Although the current findings on ANO6 function are still inconsistent, ANO6 has been found to play an important role in hemorrhagic diseases, ${ }^{9}$ bone dysplasia ${ }^{11}$ and cancer. ${ }^{13}$ However, with its role in gliomas still remaining unclear, tumor tissue was thus collected from
43 patients with glioma as well as their corresponding normal paracancerous tissues, and under the help of the techniques of RT-PCR, Western blot and immunohistochemistry, it can basically verify that the expression of ANO6 is significantly higher in glioma tissues. Survival analysis has showed that the survival rate of patients of Glioma with high expression of ANO6 is immensely lower than that of patients of glioma with low expression of ANO6. We also found that ANO6 expression correlated with WHO grade and patients' age. But there was no significant correlation with patient gender. These results 
A

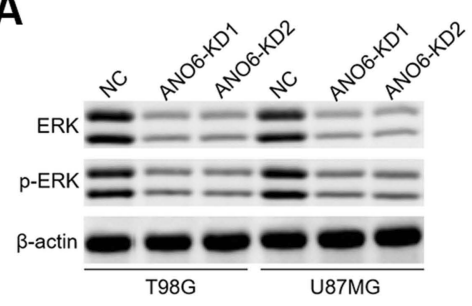

C

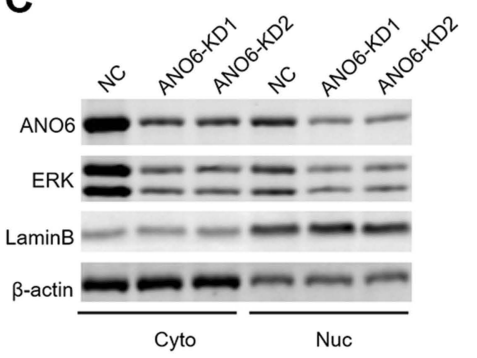

F

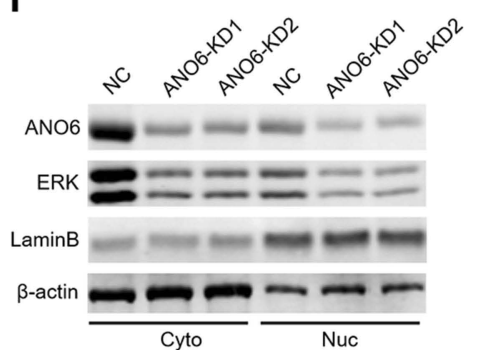

B

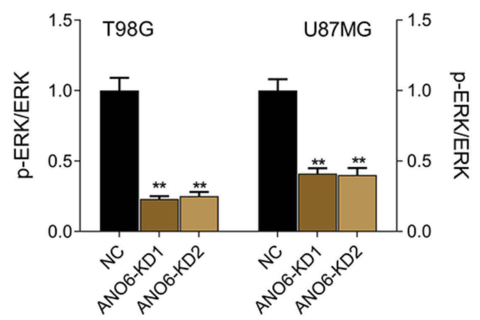

D

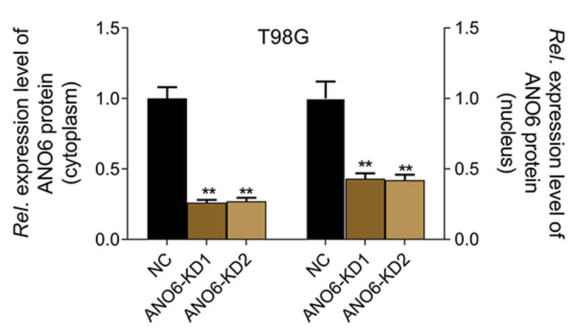

G

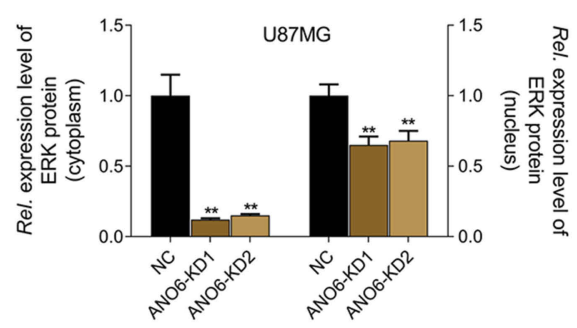

E

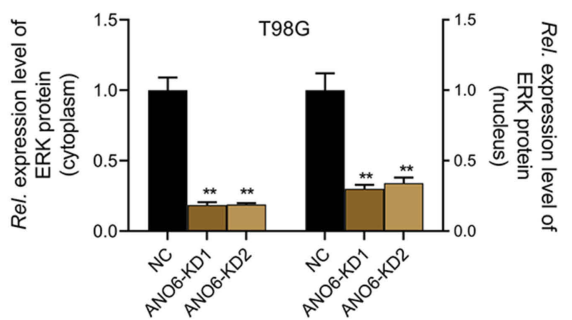

H

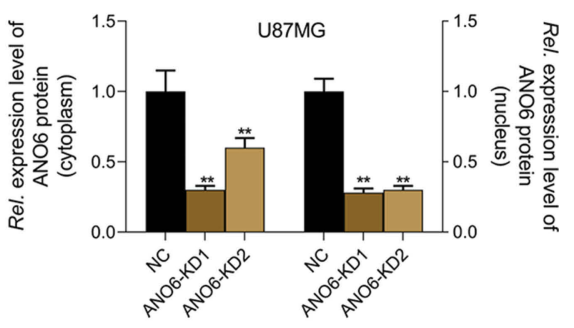

Figure 3 Knockdown of ANO6 inhibited the activation of ERK signaling. After T98G and U87MG cells transfection with ANO6-KD I or ANO6-KD2, (A and B) the protein expression level of ERK and phospho-ERK was detected by Western blot assay; (C) the protein expression levels of ANO6 and ERK were detected by Western blot assay in cytoplasm and cell nucleus of T98G cells; (D and E) Histograms were used to represent statistical results of the protein expression levels of ANO6 and ERK in cytoplasm and cell nucleus of T98G cells; (F) the protein expression levels of ANO6 and ERK were detected by Western blot assay in cytoplasm and cell nucleus of U87MG cells. (G and $\mathbf{H})$ Histograms were used to represent statistical results of the protein expression levels of ANO6 and ERK in cytoplasm and cell nucleus of U87MG cells; $\boldsymbol{\beta}$-actin was used as a load control for cytoplasm. LaminB was used as a load control for cell nucleus. Data are presented as the mean \pm standard deviation. ${ }^{* *} p<0.05$ versus ANO6-KDI group and ANO6-KD2 group.

Abbreviations: NC is negative control, Rel. is relative.

indicate that ANO6 may be play an important role in the occurrence and development of glioma. Unfortunately, univariate Cox regression analysis showed that the ANO6 expression level, age, gender and WHO stage were not related to the OS rate. Although we have not enough samples in this study to meet statistics requirements, the above results still have some clinical value for subsequent research. Next, we found that ANO6 has an obviously high expression in glioma cell lines. These results suggest that ANO6 may be a potential key gene in the development of gliomas. Therefore, the effects of ANO6 knockdown or overexpression on glioma cell proliferation and invasion were examined through MTT. Likewise, it was reflected in the results of colony formation and transwell experiments that ANO6 overexpression will substantially promote cell proliferation and invasion and ANO6 knockdown or overexpression works well on the regulation of the proliferation and invasion of glioma cells. Since ERK, being an important extracellular signal-regulated kinase, is considered as a tumor-promoting gene and is closely associated with cell proliferation, apoptosis and differentiation, ${ }^{24}$ ERK is over-activated in various cancers such as lung cancer, ${ }^{25}$ stomach cancer, ${ }^{26}$ liver cancer, ${ }^{27}$ glioma ${ }^{28}$ and breast cancer $^{29}$ to promote the development of cancer. Just as shown in some studies, ERK inhibitors can effectively inhibit the translocation of ERK by reducing phosphorylation of ERK so as to effectively treat Melanocytoma. ${ }^{30,31}$ Based on this, we apply Western Blot to detect the activation level of ERK in glioma to further explore the mechanism of ANO6 regulation of proliferation and invasion of glioma cells, revealing that ANO6 gene silence greatly reduces the phosphorylation level of ERK. Further studies showed that ANO6 knockdown enormously inhibited the expression of ERK in the cytoplasm and nucleus of glioma cells, thereby effectively decreasing ERK nuclear translocation. This suggests that ANO6 inhibition suppresses the 
A

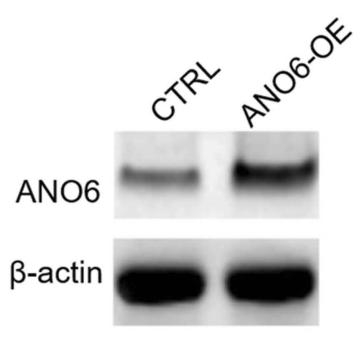

C

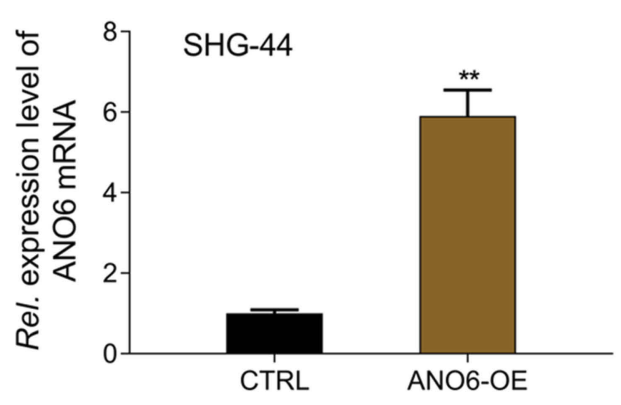

$\mathbf{E}$

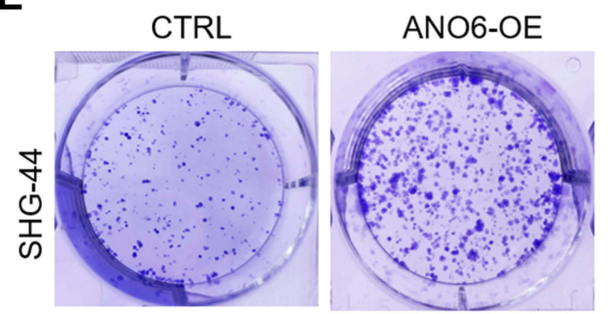

$\mathbf{F}$

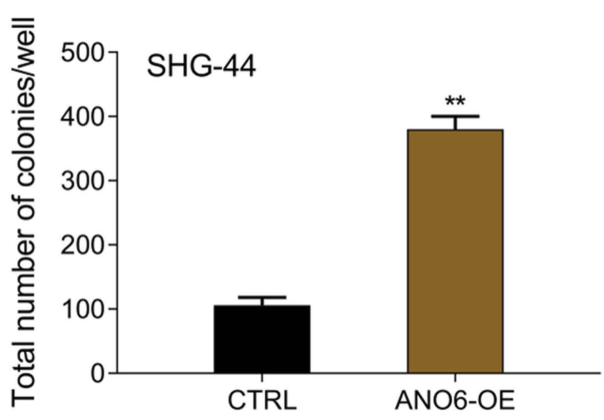

B

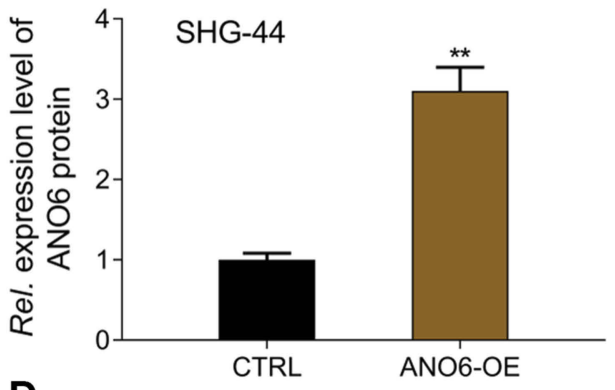

D

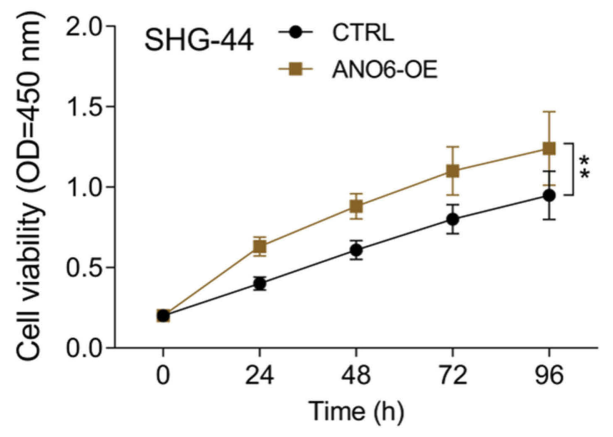

G

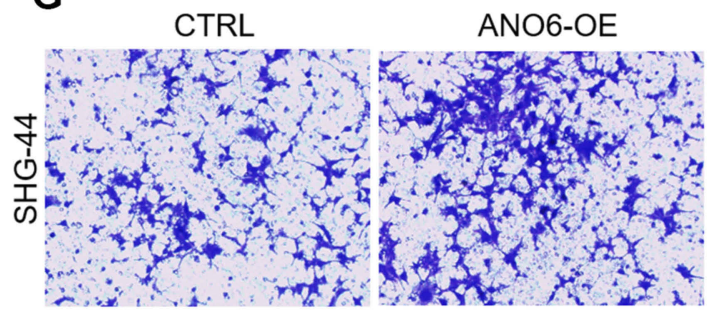

\section{H}

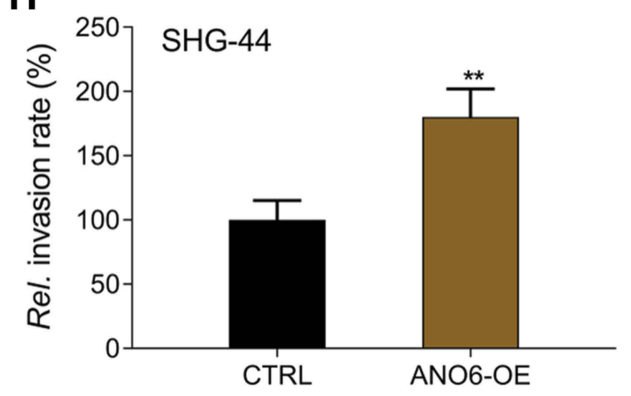

Figure 4 ANO6 overexpression promoted the proliferation and invasion of SHG-44G cells. After SHG-44 cells transfection with ANO6 plasmid, (A and B) the protein expression level of ANO6 gene was detected by Western blot assay; (C) the mRNA expression level of ANO6 gene was detected by RT-PCR assay; (D) the cell viability was detected by MTT assay; ( $\mathbf{E}$ and $\mathbf{F}$ ) the ability of cell proliferation was detected by colony formation; ( $\mathbf{G}$ and $\mathbf{H})$ the ability of invasion was detected by transwell assay. Data are presented as the mean \pm standard deviation. ${ }^{* *} p<0.05$ versus ANO6-OE group.

Abbreviations: CTRL, control, OE, overexpression, Rel. is relative.

proliferation and invasion of glioma cells by decreasing ERK nuclear translocation. To validate the findings above, we will continue examining the effect of ANO6 overexpression on proliferation and invasion of glioma cells.

RT-PCR and Western blot were used to detect the transfection effect of ANO6 overexpression plasmid transfected into SHG-44 cells. According to the MTT testing, ANO6 overexpression largely increased cell viability. Likewise, it was reflected in the results of colony formation and transwell experiments that ANO6 overexpression will substantially promote cell proliferation and invasion and ANO6 knockdown or overexpression works 


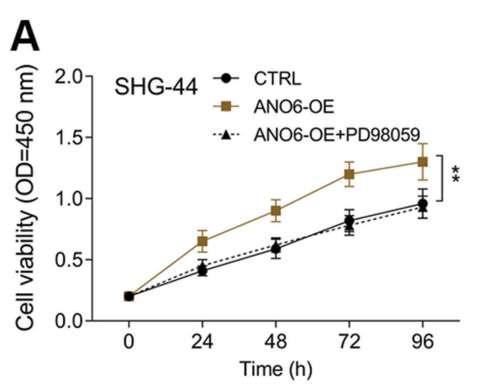

B

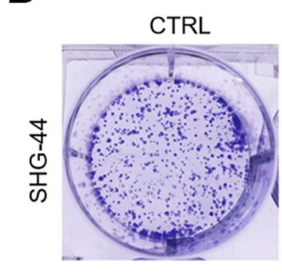

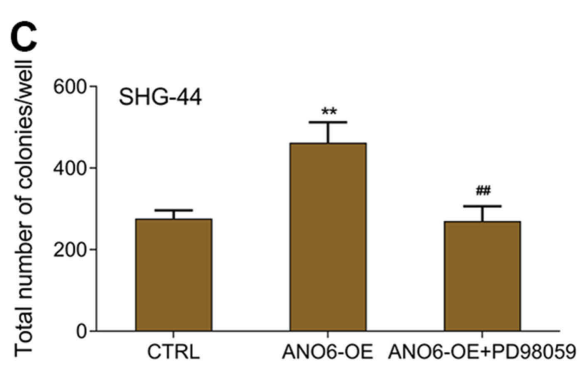

D

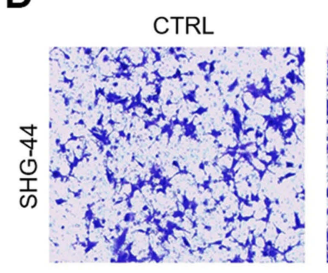

E

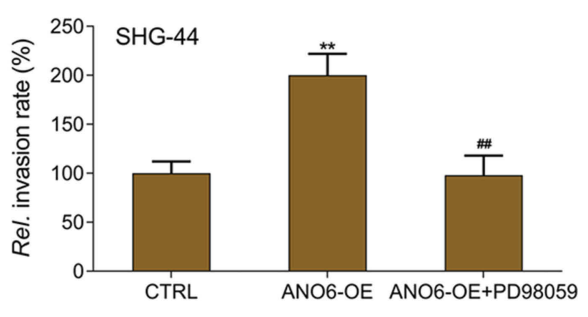

ANO6-OE

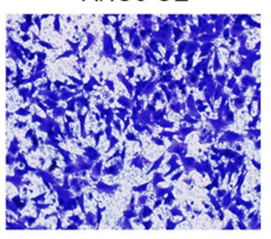

Figure 5 ERK inhibitor inhibited the proliferation and invasion of ANO6 overexpression cells. After SHG-44 cells transfection with ANO6 plasmid was treated with PD98059, (A) the cell viability was detected by MTT assay; (B and $\mathbf{C})$ the ability of cell proliferation was detected by colony formation; (D and E) the ability of invasion was detected by transwell assay. Data are presented as the mean \pm standard deviation. ${ }^{* *} p<0.05$ versus CTRL group, ${ }^{\text {\# }} p<0.05$ versus ANO6-OE group.

Abbreviations: CTRL, control, OE, overexpression, Rel., relative.

well on the regulation of the proliferation and invasion of glioma cells. In previous studies, the view that ANO6 knockdown can inhibit the phosphorylation of ERK to prevent nuclear translocation of ERK and thus curb ERK activation was reached, from which the subsequent studies will work on verification. Proven by MTT, colony formation and transwell assays, PD98059, as an ERK inhibitor, contributes to inhibiting the ANO6-mediated cell proliferation and invasion, the further result of which demonstrates that ANO6 affects the proliferation and metastasis of glioma cells by regulating the activation of ERK. In recent years, brain glioma stem cells play an important role in the treatment of glioma. In addition, brain glioma stem cells have the potential of self-renewal and multidifferentiation to cause the recurrence of glioma and the failure of radiotherapy and chemotherapy in glioma. Therefore, we plan to further study the role of ANO6 in the proliferation and self-renewal of brain glioma stem cells.

Above all, ANO6 may be a tumor-promoting gene in gliomas that regulates the proliferation and metastasis of glioma cells by promoting the nuclear translocation of ERK to activate ERK signaling.

\section{Availability of data and materials}

The datasets used and/or analyzed during the present study are available from the corresponding author on reasonable request.

\section{Ethics approval and consent to participate}

Studies were approved by the Ethics Committee of The First Hospital Affiliated to Jiamusi University, and all the samples were obtained with written informed consent and analyzed anonymously. The study was conducted in accordance with the Declaration of Helsinki.

\section{Author contributions}

All authors contributed toward data analysis, drafting and revising the paper, gave final approval of the version to be published and agree to be accountable for all aspects of the work.

\section{Disclosure}

The authors declare that they have no competing interests in this work.

\section{References}

1. Omuro A, DeAngelis LM. Glioblastoma and other malignant gliomas: a clinical review. Jama. 2013;310(17):1842-1850. doi:10.1001/ jama.2013.280319

2. Nayak L, Reardon DA. High-grade gliomas. Continuum (Minneapolis, Minn). 2017;23(6, Neuro-oncology):1548-1563. doi:10.1212/ CON.0000000000000554

3. Schiff D. Low-grade gliomas. Continuum (Minneapolis, Minn). 2017;23(6, Neuro-oncology):1564-1579. doi:10.1212/CON.0000000000000537

4. Wang Z, Terakawa $\mathrm{Y}$, Goto $\mathrm{H}$, et al. Glioblastoma in long-term survivors of acute lymphoblastic leukemia: report of two cases. Pediatr Int. 2016;58(6):520-523. doi:10.1111/ped.12843 
5. Le AP, Huang Y, Pingle SC, et al. Plexin-B2 promotes invasive growth of malignant glioma. Oncotarget. 2015;6(9):7293-7304. doi:10.18632/oncotarget.3421

6. Yu K, Duran C, Qu Z, Cui -Y-Y, Hartzell HC. Explaining calciumdependent gating of anoctamin-1 chloride channels requires a revised topology. Circ Res. 2012;110(7):990-999. doi:10.1161/CIRCR ESAHA.112.264440

7. Yang H, Kim A, David T, et al. TMEM16F forms a Ca2+-activated cation channel required for lipid scrambling in platelets during blood coagulation. Cell. 2012;151(1):111-122. doi:10.1016/j.cell.2012.07.036

8. Kunzelmann K, Tian Y, Martins JR, et al. Anoctamins. Pflugers Archiv. 2011;462(2):195-208. doi:10.1007/s00424-011-0975-9

9. Kmit A, van Kruchten R, Ousingsawat J, et al. Calcium-activated and apoptotic phospholipid scrambling induced by Ano6 can occur independently of Ano6 ion currents. Cell Death Dis. 2013;4(4):4e611. doi:10.1038/cddis.2013.135

10. Tsutsumi S, Kamata N, Vokes TJ, et al. The novel gene encoding a putative transmembrane protein is mutated in gnathodiaphyseal dysplasia (GDD). Am J Hum Genet. 2004;74(6):1255-1261. doi:10.1086/421527

11. Ehlen HW, Chinenkova M, Moser M, et al. Inactivation of anoctamin-6/Tmem16f, a regulator of phosphatidylserine scrambling in osteoblasts, leads to decreased mineral deposition in skeletal tissues. J Bone Miner Res. 2013;28(2):246-259. doi:10.1002/jbmr.1751

12. Damek-Poprawa M, Golub E, Otis L, Harrison G, Phillips C, BoeszeBattaglia K. Chondrocytes utilize a cholesterol-dependent lipid translocator to externalize phosphatidylserine. Biochemistry. 2006;45 (10):3325-3336. doi:10.1021/bi0515927

13. Fan CW, Chen CY, Chen KT, et al. Blockade of phospholipid scramblase 1 with its $\mathrm{N}$-terminal domain antibody reduces tumorigenesis of colorectal carcinomas in vitro and in vivo. J Transl Med. 2012;10 (1):10254. doi:10.1186/1479-5876-10-233

14. Kato U, Inadome H, Yamamoto M, Emoto K, Kobayashi T, Umeda M. Role for phospholipid flippase complex of ATP8A1 and CDC50A proteins in cell migration. J Biol Chem. 2013;288(7):4922-4934. doi:10.1074/jbc.M112.402701

15. Jacobsen KS, Zeeberg K, Sauter DR, Poulsen KA, Hoffmann EK, Schwab A. The role of TMEM16A (ANO1) and TMEM16F (ANO6) in cell migration. Pflugers Archiv. 2013;465(12):1753-1762. doi:10.1007/ s00424-013-1315-z

16. Godse NR, Khan N, Yochum ZA, et al. TMEM16A/ANO1 inhibits apoptosis via downregulation of bim expression. Clin Cancer Res. 2017;23(23):7324-7332. doi:10.1158/1078-0432.CCR-17-1561

17. Yu Z, Ye S, Hu G, et al. The RAF-MEK-ERK pathway: targeting ERK to overcome obstacles to effective cancer therapy. Future Med Chem. 2015;7(3):269-289. doi:10.4155/fmc.14.143

18. Shindo Y, Iwamoto K, Mouri K, et al. Conversion of graded phosphorylation into switch-like nuclear translocation via autoregulatory mechanisms in ERK signalling. Nat Commun. 2016;7:10485. doi: $10.1038 /$ ncomms 10485
19. Berti DA, Seger R. The nuclear translocation of ERK. Methods Mol Biol. 2017;1487:175-194.

20. Lyros O, Lamprecht AK, Nie L, et al. Dickkopf-1 (DKK1) promotes tumor growth via Akt-phosphorylation and independently of Wntaxis in barrett's associated esophageal adenocarcinoma. Am J Cancer Res. 2019;9(2):330-346.

21. Palumbo P, Lombardi F, Siragusa G, et al. Involvement of NOS2 activity on human glioma cell growth, clonogenic potential, and neurosphere generation. Int $J$ Mol Sci. 2018;19(9):2801. doi:10.3390/ijms19092801

22. Ousingsawat J, Wanitchakool P, Schreiber R, Kunzelmann K. Contribution of TMEM16F to pyroptotic cell death. Cell Death Dis. 2018;9(3):300. doi:10.1038/s41419-018-0373-8

23. Schenk LK, Ousingsawat J, Skryabin BV, Schreiber R, Pavenstädt H, Kunzelmann K. Regulation and function of TMEM16F in renal podocytes. Int $J$ Mol Sci. 2018;19(6):1798. doi:10.3390/ijms 19061798

24. Jin X, Sun J, Yu B, et al. Daidzein stimulates osteogenesis facilitating proliferation, differentiation, and antiapoptosis in human osteoblastlike MG-63 cells via estrogen receptor-dependent MEK/ERK and PI3K/Akt activation. Nutr Res. 2017;42:20-30. doi:10.1016/j. nutres.2017.04.009

25. Yang Y, Zhao J, Hao D, et al. Increased SPK1 expression promotes cell growth by activating the ERK1/2 signaling in non-small-cell lung cancer. Anticancer Drugs. 2019. doi:10.1097/CAD.0000000000000 733

26. Zhu YM, Li Q, Gao XZ, et al. C14orf159 suppresses gastric cancer cells' invasion and proliferation by inactivating ERK signaling. Cancer Manag Res. 2019;11:1717-1723. doi:10.2147/CMAR. S176771

27. Li Z, Li Z, Wang L, et al. ZCCHC13-mediated induction of human liver cancer is associated with the modulation of DNA methylation and the AKT/ERK signaling pathway. J Transl Med. 2019;17(1):108. doi:10.1186/s12967-019-1973-5

28. Mu P, Liu K, Lin Q, et al. Sirtuin 7 promotes glioma proliferation and invasion through activation of the ERK/STAT3 signaling pathway. Oncol Lett. 2019;17(2):1445-1452. doi:10.3892/ol.2018.9800

29. Cai M, Liang X, Sun X, et al. Nuclear receptor coactivator 2 promotes human breast cancer cell growth by positively regulating the MAPK/ERK pathway. Front Oncol. 2019;9:164. doi:10.3389/ fonc. 2019.00164

30. Blair CM, Walsh NM, Littman BH, et al. Targeting B-Raf inhibitor resistant melanoma with novel cell penetrating peptide disrupters of PDE8A - C-Raf. BMC Cancer. 2019;19(1):266. doi:10.1186/s12885019-5699-9

31. Savoia P, Fava P, Casoni F, Cremona O. Targeting the ERK signaling pathway in melanoma. Int J Mol Sci. 2019;20(6):1483. doi:10.3390/ ijms20061483
OncoTargets and Therapy

\section{Publish your work in this journal}

OncoTargets and Therapy is an international, peer-reviewed, open access journal focusing on the pathological basis of all cancers, potential targets for therapy and treatment protocols employed to improve the management of cancer patients. The journal also focuses on the impact of management programs and new therapeutic

Submit your manuscript here: https://www.dovepress.com/oncotargets-and-therapy-journa agents and protocols on patient perspectives such as quality of life, adherence and satisfaction. The manuscript management system is completely online and includes a very quick and fair peer-review system, which is all easy to use. Visit http://www.dovepress.com/ testimonials.php to read real quotes from published authors. 